Casos Clínicos

Arch. Esp. Urol., 59, 2 (183-186), 2006

\section{PAPILOMA INVERTIDO DE LA URETRA ANTERIOR MASCULINA. PRESENTACIÓN DE UN CASO Y BREVE REVISIÓN DE LA LITERATURA.}

\author{
Otto Ochoa Undargarain, José Alberto Hermida Pérez' \\ y Johannes Ochoa Montes de Oca
}

Hospital Provincial Manuel Ascunce. Domenech. Camagüey. Cuba.

Servicio de Urgencias de Los Llanos de Aridene?. Tenerife. España.

Instituto Superior de Ciencias Médicas de Camagüey².

Cuba.

Resumen.- OBJETIVO: Presentar un caso clínico de un paciente masculino con un papiloma invertido de la uretra anterior.

MÉTODO/RESULTADO: Se trata de un paciente varón, de 40 años de edad, con antecedentes de salud, que acude a consulta de Urología por presentar un cuadro de disuria y uretrorragia en 3 ocasiones. Tras la realización de un estudio previo que incluyó radiografía de uretrocistografía miccional y en la que se observó un defecto de llenado en uretra anterior, se decide realizar cistouretroscopía bajo anestesia, realizándose en este mismo acto una resección endoscópica de la tumoración que ocasionaba dicho defecto de llenado. El estudio anatomopatológico de la pieza informa un papiloma invertido uretral.
CONCLUSIONES: La localización en la uretra masculina del papiloma invertido es rara. Las manifestaciones clínicas más frecuentes son la disuria y la hematuria, aunque los situados en uretra anterior pueden provocar uretrorragia. El diagnóstico se realiza por la clínica, la ecografía, la endoscopía y la uretrocistografía miccional, en la que se puede observar un defecto de llenado a nivel de la uretra. El tratamiento consiste en la resección transuretral (RTU) de la lesión, siendo conveniente los controles endoscópicos anuales tras la cirugía.

Palabras clave: Disuria. Uretrorragia. Papiloma invertido uretral.

Summary.- OBJECTIVES: We report a clinical case of inverted papilloma of the anterior male urethra.

METHODS/RESULTS: 40-year-old male without significant past medical history presenting with dysuria and referring three episodes of urethral bleeding. Preliminary studies included voiding cystourethrogram (VCUG) which revealed a filling defect in the anterior urethra. Urethrocystoscopy was performed under anesthesia, with resection of the tumor. Pathologic study reported a urethral inverted papilloma.

CONCLUSIONS: Inverted papillomas of the male urethra are rare. The most frequent clinical manifestations are dysuria and hematuria, although they may present with urethral bleeding when located in the anterior urethra. Diagnosis is based on clinical symptoms, ultrasound, endoscopy and VCUG which enable detection of urethral filling defects. The treatment of choice is transurethral resection of the lesion. Annual endoscopic control is recommended after surgery.

Keywords: Dysuria. Urethral bleeding. Urethral inverted papilloma

\title{
INTRODUCCIÓN
}

Un papiloma invertido es una lesión proliferativa benigna causada por una inflamación crónica o por una obstrucción crónica del tracto vesical de salida. Con mayor frecuencia se observa en el trígono y en el cuello vesical en hombres con prostatismo (1), aunque se han descrito casos de papilomas invertidos en las diferentes porciones de las vías urinarias y en ambos sexos como son en la pelvis renal (2), el uréter (3), la ya mencionada localización vesical (4), la uretra prostática (5) y la uretra femenina (6) y también se han descrito papilomas invertidos vesicales múltiples (7). 
El papiloma invertido es una entidad poco frecuente, es un tumor del tracto urinario inferior, que su incidencia exacta no se conoce, aunque se estima en el $2,2 \%$ de todas las lesiones de la vía urinaria, siendo la localización más habitual en el cuello vesical $(44,7 \%)$, el trígono $(36,8 \%)$, y rara la localización en uretra prostática $(3,5 \%)(5,6)$.

Microscópicamente las papilas se proyectan hacia el estroma fibrovascular de la vejiga en lugar de protruir en la luz vesical. La lesión por lo general está recubierta por una capa de urotelio normal y pueden contener áreas de cistitis quística o metaplasia escamosa. Se pueden observar dos tipos histológicos, el trabecular originado en una proliferación de las células basales, y el glandular que representa una forma de cistitis glandular que se origina en las células intermedias y como tal es considerado una lesión con potencial neoplásico (8).

Clínica y macroscópicamente es fácilmente confundido con un carcinoma de urotelio, pero por su histología tan característica se ha considerado como una lesión con entidad propia, cuya histopatogenia es aún muy discutida (5). Esta entidad debe su nombre a Potts y Hirst quienes en 1963 la describieron (9), aunque anteriormente ya se habían descrito como pólipo adenomatoide de la mucosa vesical (10).

A pesar de ser considerada en un principio como una lesión benigna, se han publicado casos de recidiva de papiloma invertido (8), así como de asociación a carcinoma transicional tanto de forma sincrónica como asincrónica (11-13)

\section{CASO CLÍNICO}

Paciente varón, de 40 años de edad, con antecedentes de salud. Acude a consulta de Urología por presentar disuria y 3 episodios de uretrorragia. Al examen físico las mucosas son húmedas y normocoloreadas, la auscultación cardio-respiratoria es normal, el abdomen es negativo, fosas lumbares libres, el tacto rectal, los genitales externos y las regiones inguinales son normales. Se le realiza ecografía urológica que es normal. La cistouretroscopía es normal, sólo notándose un leve resalto al paso del cistoscopio por uretra anterior.

Se le realiza radiografía de uretrocistografía miccional donde se observa un defecto de llenado a nivel de la uretra anterior (Figura 1). Por todo lo anterior decidimos realizar un estudio endoscópico bajo anestesia, detectándose una tumoración polipoide, de color rojiza, bien delimitada, por lo que se decide resección endoscópica de la misma.

El resultado anatomopatológico de la pieza quirúrgica fue un papiloma invertido de uretra anterior. Se da de alta por mejoría, evolucionando satisfactoriamente con desaparición de la disuria y la uretrorragia.

\section{DISCUSIÓN}

Las diferentes edades de aparición del papiloma invertido varían según las diversas publicaciones. Se plantea que la edad de mayor incidencia se sitúa entorno a los 60 años, habiéndose descrito un caso en un niño de 14 años (14). Marquez Moreno y cols. (2001) afirman que los papilomas invertidos de las vías urinarias suelen afectar a adultos de 24 a 77 años de edad, con un pico máximo entre los 50 y los 70 años (7), Valero Puerta y cols. (1995) publican una casuística de 12 pacientes afectos de esta entidad cuyas edades oscilan entre los 36 a los 79 años de edad (15), la edad de aparición en nuestro paciente fue a los 40 años.

El hallazgo de papilomas invertidos en la uretra masculina constituye una rareza clínica, lo que queda reflejado en las diferentes publicaciones que hemos revisado 15 , $6,15-17)$.

Desde el punto de vista de sus manifestaciones clínicas, estas dependerán de su localización en el tracto urinario, siendo los episodios de hematuria autolimitada macroscópica y los síntomas de prostatismo (disuria, polaquiuria, etc.) las más frecuentes en aquellos papilomas invertidos localizados en la vejiga y la uretra posterior (uretra prostática), hay un caso publicado de un papiloma invertido de uretra femenina, localizado sobre el labio posterior del meato uretral externo, que se diagnosticó al consultar la paciente por presentar metrorragia postmenopáusica, resultando ser el papiloma la causa del sangramiento; los localizados en las porciones más altas de las vías urinarias (pelvis y uréter) tendrán a la hematuria como su principal síntoma $(2,3,18)$.

Nuestro paciente con un papiloma localizado en la uretra anterior se manifestó con síntomas de disuria y

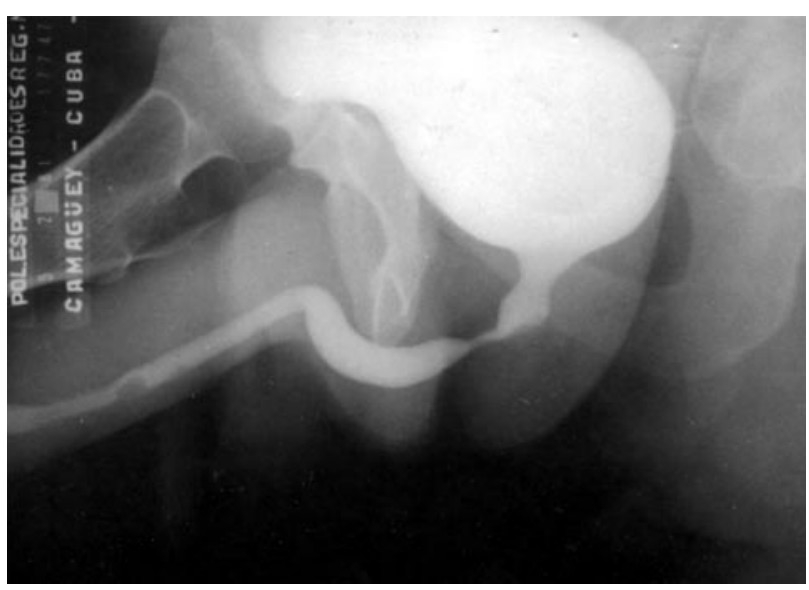

FIGURA 1. RX uretrocistografía miccional en la que se puede observar un defecto de llenado a nivel de la uretra anterior. 
uretrorragia. Desde el punto de vista de los exámenes complementarios en los que nos auxiliamos para realizar el diagnóstico, la ecografía, la endoscopía y los exámenes contrastrados de las vías urinarias (urografía excretora, uretrocistografía miccional y retrógrada y pielografía ascendente) constituyen exploraciones de gran importancia para realizar el diagnóstico de esta entidad, siendo el examen anatomopatológico de la pieza lo que confirma el mismo, pudiéndose realizarse también estudios inmunohistoquímicos (Ki-67/Mib-1), así como citometría de imagen para determinar la ploidía del $A D N$ con el objetivo constatar si estas lesiones tienen actividad proliferativa $(7,15)$.

En la Ecografía se suele observar una lesión excrecente o polipoide, ecográficamente homogénea y en la endoscopía se ven como una formación polipoide, pediculada, con una base de implantación, cubierta por mucosa urotelial normal $(5,7)$. Los estudios radiológicos contrastados de las vías urinarias nos mostrarán un defecto de llenado en el sitio donde se encuentre ubicada la lesión (15).

En nuestro paciente utilizamos la endoscopía, la uretrocistografía miccional y retrógrada y el examen anatomopatológico del espécimen de resección transuretral (RTU) para llegar a establecer el diagnóstico. El tratamiento utilizado por los diversos autores consultados por nosotros ha sido la RTU de la lesión con el posterior estudio anatomopatológico de la muestra obtenida para la confirmación histológica $(5,7,10,16)$.

Aunque se dice que son lesiones proliferativas benignas, dado su aspecto citohistológico en el estudio microscópico y a la historia natural de la lesión $(1,7)$, algunos autores, apoyados en la asociación del papiloma invertido con neoplasias vesicales malignas y en el hallazgo de índices proliferativos elevados en algunas de estas lesiones, tienden a clasificarlas como tumores de bajo grado de agresividad por lo que recomiendan un largo seguimiento de los pacientes (19), proponiéndose un seguimiento endoscópico anual tras el diagnóstico (4, 10,12 ) e incluso llegándose a defender la instauración de quimioterapia endocavitaria como pauta terapéutica después de la RTU de la lesión (12).

Valero Puerta (1995) (15) en su casuística, de un total de 12 pacientes con papilomas invertidos de las vías urinarias, detecta que en 5 de ellos había asociación con carcinoma de células transicionales (CCT) y concluye afirmando, tras analizar los resultados obtenidos en sus estudios de cuantificación de la expresión del antígeno $\mathrm{Ki}-67$, realizando un marcaje inmunohistoquímico de los tumores con anticuerpor Mib-1, que el papiloma invertido se ha de tratar como un Ca. papilar transicional superficial, ya que puede recidivar 0 asociarse a Ca. transicional. Las lesiones con una elevada actividad proliferativa, que es facilmente evaluada con la cuantificación del antígeno Ki-67, generalmente evolucionarán de modo desfavorable.
Otros autores como Marquez Moreno y cols. (2001) (7) también realizan estudios de determinación de la actividad proliferativa de estas lesiones, mediante la cuantificación de Ki-67/Mib-1, siendo esta muy baja en su casuística y determinan la ploidía de ADN mediante citometría de imagen, resultando diploides sus dos lesiones, lo que concuerda con la evolución benigna de su caso clínico, con una ausencia de recidivas hasta la actualidad. Asano y cols. (2003) presentan una casuística de 48 pacientes afectos de papilomas invertidos de las vías urinarias, en edades de 24 a 82 años, reportando 3 casos asociados a CCT y recidivas post RTU en 3 pacientes (a los cinco meses, al año y cuatro meses y a los dos años y seis meses), estos autores concluyen afirmando que existen dos formas de papilomas invertidos, una benigna y otra con potencial maligno (20).

Nosotros compartimos la opinión de otros autores los que plantean que sería suficiente la RTU de la lesión, descartando la asociación con carcinoma de urotelio, y el seguimiento endoscópico anual $(5,7)$.

\section{BIBLIOGRAFÍA Y LECTURAS

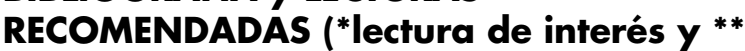 lectura fundamental)}

1. DEMEESTER, L.J.; FARROW, G.M.; UTZ, D.C.: "Inverted papilloma of the urinary bladder". Cancer, 36: 505, 1975.

2. BAGLEY, D.H.; McCUE, P.; BLACKSTONE, A.S.: "Inverted papilloma of renal pelvis: flexible ureteroscopic diagnosis and treatment”. Urol., 36: 336, 1990.

3. CASTILLO, J.M.; SANTIAGO, A.; RUIZ, J. L. y cols.: "Papiloma invertido del uréter". Arch. Esp. Urol., 44: 1193, 1991.

4. GARMENDIA, J.C.; LÓPEZ, J.A.; ARROCENA, F. y cols.: "Papiloma invertido de vejiga. A propósito de dos casos". Arch. Esp. Urol., 43: 1013, 1990.

5. VESA LLANES, J.; DOMINGO FERRERONS, R.; MUNTANE OBRADOS, M. J. y cols.: "Papiloma invertido de la uretra prostática. Consideraciones histopatogénicas”. Arch. Esp. Urol., 47: 1022, 1994.

6. VIGURI DÍAZ, A.; CORDOBA ITURRIAGATOITIA, A.; MILLAN, J.A .: "Papiloma invertido de uretra femenina". Arch. Esp. Urol., 48: 954, 1995.

**7. MARQUEZ MORENO, A.J.; JULVE VILLALTA, E.; ALONSO DORREGO, J.M., y cols.: "Papilomas invertidos vesicales múltiples”. Arch. Esp. Urol., 54: 692, 2001.

8. KUNZE, E.; SCHAUER, A.; SCHMITT, M.: "Histology and histogenesis of two different types of inverted urothelial papillomas". Cancer, 51: 348, 1983.

9. POTTS, I.F.; HIRST, E.: "Inverted papilloma of the bladder". J. Urol., 90: 175, 1963. 
*10. PÉREZ- CASTRO, E.; GAMALLO, G.; AVELLANA, J. A. y cols.: "Papilomas invertidos de vejiga. Presentación de tres casos y revisión de la literatura". Arch. Esp. Urol., 33: 107, 1980.

11. CALAHORRA FERNANDEZ, F. J.; USERA, G.; RELEA, F.: "Papiloma de pelvis renal asociado con carcinoma de células transicionales de vejiga: aportación de un caso y revisión de la literatura". Actas Urol. Esp., 17: 689, 1993.

12. RENFER, L.G.; KELLEY, J.; BELVILLE, W.D.: "Inverted papilloma of the urinary tract: histogenesis, recurrence and associated malignancy". J. Urol., 140: 832, 1988.

13. MULKENS, T.H.; VEREYCKEN, H.A.; VAN NUETEN, J. C. y cols.: "Bilateral ureteral inverted papillomas in association with bladder carcinoma: a case report and review of the literature". Urol. Radiol., 12: 154, 1990.

14. FRANCIS, R.R.: "Inverted papilloma in a 14 yearsold male". Br. J. Urol., 51: 327, 1979.

**15. VALERO PUERTA, J.A.; REDONDO MARTINEZ, E.; JIMENEZ GONZALEZ, C. y cols.: "Papiloma invertido transicional: expresión del antígeno nuclear Ki-67 como factor pronóstico". Arch. Esp. de Urol., 48: 887, 1995.

16. OJEA, A.: "Papiloma invertido de uretra". Act. Urol. Esp., 17: 193, 1993.

17. PELliCE, C.; CARRETERO, P.; GIL VERNET, J.M.: "Papilomas invertidos del tracto urinario inferior. Consideraciones histológicas, terapéuticas y pronósticas". Arch. Esp. Urol., 40: 165, 1987.

18. KYRIAKOS, M.; ROYCE, R.K.: "Multiple simultaneous inverted papillomas of the upper urinary tract". Cancer, 63: 368, 1989.

19. FERRERO DORIA, R.; GUZMAN MARTINEZVALLS, P.L.; MORGA EGEA, J.P. y cols.: "Inverted papilloma: presentation of 5 cases and review of the Spanish literature.” Actas Urol. Esp., 22: 131, 1998.

**20. ASANO; KOJI; MIKI y cols.: "Clinical studies the inverted papillomas of the urinary tract: report of 48 cases and rewiev of the literature". J. Urol., 170: 1209, 2003.
Casos Clínicos

Arch. Esp. Urol., 59, 2 (186-189), 2006

\section{TUMOR FIBROSO SOLITARIO DE LA TÚNICA VAGINAL.}

Marta García Torrelles, Jose Ramón Beltrán Armada, Ignacio Santolaya García, Víctor Carrascosa Lloret, Miguel Tarín Planes y Carlos San Juan De Laorden.

Servicio de Urología. Hospital Universitario Dr. Peset. Valencia. España.

Resumen.- OBJETIVO: Presentamos un caso de tumor fibroso solitario de la túnica vaginal, una lesión paratesticular de naturaleza benigna.

MÉTODO: Se llevó a cabo una exploración quirúrgica con biopsia peroperatoria, y excisión de la lesión, dejando indemnes teste y epidídimo.

RESULTADOS: la biopsia intraoperatoria reveló la presencia de tejido fibroso sin celularidad maligna. El diagnóstico histopatológico de la pieza quirúrgica fue de tumor fibroso solitario de la túnica vaginal. El paciente está asintomático y libre de enfermedad un año tras el tratamiento.

CONCLUSION: Casi todas las masas testiculares son malignas, pero hay que ser cautos ante los hallazgos ecográficos y quirúrgicos de una lesión paratesticular de probable naturaleza benigna, con el fin de evitar una orquiectomía radical no indicada.

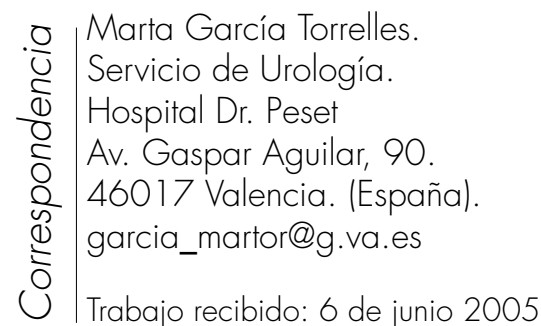

